\title{
Der Nutzen wird nicht quantifiziert
}

Jürg Nadiga, Thomas Kroner ${ }^{b}$

a Präsident der Schweizerischen Gesellschaft für Medizinische Onkologie

b Ehemaliger Leiter der Arbeitsgruppe OLU der SGMO und der SGV, Facharzt für Medizinische Onkologie und Innere Medizin
In der SÄZ vom 30.10.2013 orientieren Beat Seiler et al. über die Erfahrung der Helsana mit dem MediScore-Modell im Off Label Use [1]. Dieses Modell benutzt ein Punktesystem und beurteilt aufgrund von Studiendaten, ob der Nutzen einer Behandlung genügend gross ist, um sie ausserhalb der Spezialitätenliste zulasten der Krankenversicherung zu vergüten. Das Modell erlaubt aber keine quantitative Nutzenbewertung: Der Score für eine Therapie ist gleich hoch, ob sie zu einer Lebensverlängerung von drei Jahren oder nur von sechs Monaten führt. Zudem ziert und transparent in eine Vergütung überführt, wäre da zielführend.

Da die ausgehandelten Vergütungen zu den einzelnen Indikationen nicht offengelegt werden, bleibt die Vermutung, dass der Off Label Use (OLU) für die Pharmafirmen weiterhin attraktiver ist als die ordentliche Registrierung einer neuen Indikation. Statt zu fordern, in der Schweiz solle nur mit einem Modell gearbeitet werden, sollten vielmehr die Vergütungen $\mathrm{zu}$ den einzelnen Indikationen offengelegt werden. So lange dies nicht geschieht, birgt das MediScore-

\section{«Lediglich ein Modell, das den Nutzen quantifiziert und transparent in eine Vergütung überführt, wäre da zielführend.»}

kann in diesem Modell auch ein sehr bescheidener Nutzen - beispielsweise eine Verlängerung nur des progressionsfreien Überlebens um lediglich acht Wochen - bereits dazu führen, dass KVV Art. 71 als erfüllt betrachtet und die Kostenübernahme empfohlen wird.

Im Helsana-Modell werden Behandlungen, die die Bedingungen von Art. 71 KVV für die Kostenübernahme erfüllen, aufgrund der Punktezahl semiquantitativ in zwei Nutzenkategorien eingeteilt. Wie diese in eine Vergütung überführt werden, wird nicht kommuniziert. Der Bundesgerichtsentscheid zu Myozym und der Art. $71 \mathrm{KVV}$ verlangen aber eine Korrelation von Vergütung und Nutzen. Diese Bedingung erfüllt das Helsana-Modell mit lediglich zwei Kategorien u. E. nicht.

Weder die Abstufung der Vergütung entsprechend den beiden Kategorien noch die Höhe der Vergütung werden kommuniziert. Bei fehlender Transparenz ist somit auch nicht nachvollziehbar, ob die von den Versicherern ausgehandelte Vergütung dem Gebot der Verallgemeinerbarkeit Rechnung trägt, die das Bundesgericht im Myozymentscheid fordert [2]. Der hohe Preis der Top 20 Medikamente und der in den Studien oft bescheidene Nutzen von solchen teuren, zielgerichteten Therapien lassen zweifeln, ob die verhandelten Vergütungen tatsächlich dem Gebot der Verallgemeinerbarkeit genügen. Somit eignet sich das Mediscore-Modell nicht, den Bundesgerichtsentscheid zu Myozym und damit Art. 71 KVV umzusetzen. Lediglich ein Modell, das den Nutzen quantifi-
Modell die Gefahr in sich, eine Vergütungspraxis zu legitimieren, die den Willen des Gesetzgebers umgeht, auch im OLU nur verallgemeinerbare Vergütungen zu bezahlen. Diese Industriefreundlichkeit des Modells ist nicht weiter verwunderlich, wurde es doch in Zusammenarbeit mit den Herstellern erarbeitet.

Die evidenzbasierte Nutzenbewertung (EBN) der SGMO [3] weist die oben beschriebenen Mängel nicht auf. Sie legt den Nutzen aufgrund von Studien-

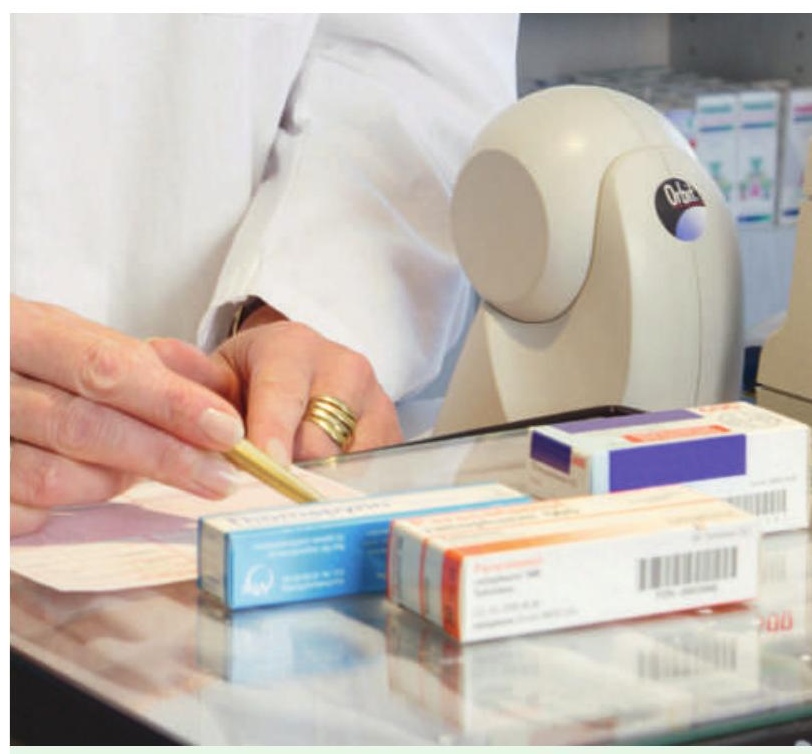

Die Vergütung von Medikamenten sollte mit ihrem Nutzen korrelieren. 
daten quantitativ fest. Der Nutzen setzt sich aus den zwei zentralen Parametern Lebensverlängerung und Lebensqualitätsverbesserung zusammen. Er wird linear in eine Vergütung übergeführt. Eine mögliche Basis für die Festlegung der Vergütung wird vorgeschlagen. Damit wäre die jährliche Vergütung für Glivec bereits nach der Erstpublikation etwa gleich hoch gewesen wie die heutigen Jahreskosten.

Die Probleme im OLU lassen sich wegen der verschiedenen gesetzlichen Rahmenbedingungen wahrscheinlich nur über eine Tarifvereinbarung zwischen Industrie, Kostenträgern und Ärzten lösen. Das in einer solchen Tarifvereinbarung verwendete Modell sollte eine evidenzbasierte quantitative Nutzenbewertung linear in eine Vergütung überführen.

\section{Literatur}

1 Seiler B, Fries R, Honegger H. Medikamente im Offlabel-Use. Schweiz Ärztezeitung. 2013;94(44):1680-3.

2 Für die Heilung eines einzelnen Kranken könne nicht jeder Preis bezahlt werden, weil sonst die Mittel an einem anderen Ort fehlten (Utilitaristisches Kriterium). Kriterium für die Beschaffung und die Verteilung staatlicher Mittel sei die Verteilungsgerechtigkeit. Rechtsgleichheit setze Verallgemeinerungsfähigkeit voraus. Verallgemeinerungsfähig sei aber nur, was allen, die sich in der gleichen Situation befinden, in gleicher Weise angeboten werden kann. Ohne besondere Rechtfertigung sei es mit der Rechtsgleichheit und der Gleichwertigkeit aller Menschen nicht vereinbar, einzelnen Versicherten Leistungen zu erbringen, die anderen Versicherten in gleicher Lage nicht erbracht werden können. Gemäss diesem Prinzip berechnet das BG die Therapiejahreskosten. Beim M. Pompe begrenzt die Atemnot wegen einer Schwäche der Atemmuskulatur die Mobilität der Kranken. In der Schweiz gebe es 2,5\% Einwohner, die wegen einer schweren COPD gleich eingeschränkt seien, wie die Patientin mit M. Pompe. Müsste ihnen allen eine gleich teure Therapie zugänglich gemacht werden, wäre allein für diese fiktive Behandlung 1,5-mal das Gesamtbudget des öffentlichen Gesundheitswesens aufzuwenden. Damit sei die Behandlung nicht verallgemeinerungsfähig.

3 Nadig J. Evidenzbasierte Nutzenbewertung: ein Modell zur Vergütung des Off Label Use in der Onkologie. Schweiz Ärztezeitung. 2012;93(14/15):552. 